\title{
Advanced Sample Preparation Techniques Using Broad Ar Ion Beam for Optimum EBSD Acquisition
}

\author{
N. Erdman*, K. Ogura**, and R. Campbell* \\ * JEOL USA Inc, 11 Dearborn Rd, Peabody, MA \\ ** JEOL Ltd, Tokyo 196-8558, Japan
}

Electron Backscatter Diffraction (EBSD) is a powerful technique capable of characterizing extremely fine grained microstructures in a Scanning Electron Microscope (SEM). Electron Back Scatter Patterns (EBSPs) are generated near the sample surface, typically from a depth in the range $10-50 \mathrm{~nm}$. Consequently, EBSD requires that the surface is crystallographically damagefree on a nanometer scale. For this reason, sample preparation is critical to achieving high EBSP quality, which in turn, is an important prerequisite to be able to maximize acquisition speed.

Several years ago JEOL has developed and introduced a cross-section sample preparation device for SEM (the Cross-section polisher or CP) that addresses some of the issues involved with other sample preparation methods like mechanical polishing or microtomy. CP can easily prepare an SEM cross section and can preserve nanometer-level fine structures. CP uses a high-energy Ar ion beam to irradiate the surface of the specimen masked by a shielding plate, while the surface region which is not masked by the shielding plate is etched (Fig. 1). This method provides two advantages: a) a clean cross section can be obtained, which has less distortion or altered layers due to milling vs. a sample prepared by conventional polishing methods; b) a wider-region cross section can be prepared as compared to FIB with less damage. In addition to a standard specimen configuration, the instrument also features a newly redesigned rotation stage that can polish up to 1 in wide mounts.

We will show example of various materials prepared by $\mathrm{CP}$ and subsequently analyzed with EBSD. The examples include thin films for solar applications (Fig. 2), nano-grained metals and ceramics. We will also show how highly heterogeneous and otherwise difficult specimens, for example shale, can be easily prepared without any additional embedding procedures and then analyzed via EBSD.

References:

N. Erdman, AM\&P, vol. 6, 33-35 (2006)

S. Asahina et al, EMC 2008, I3 (1), 525-526 (2008) 


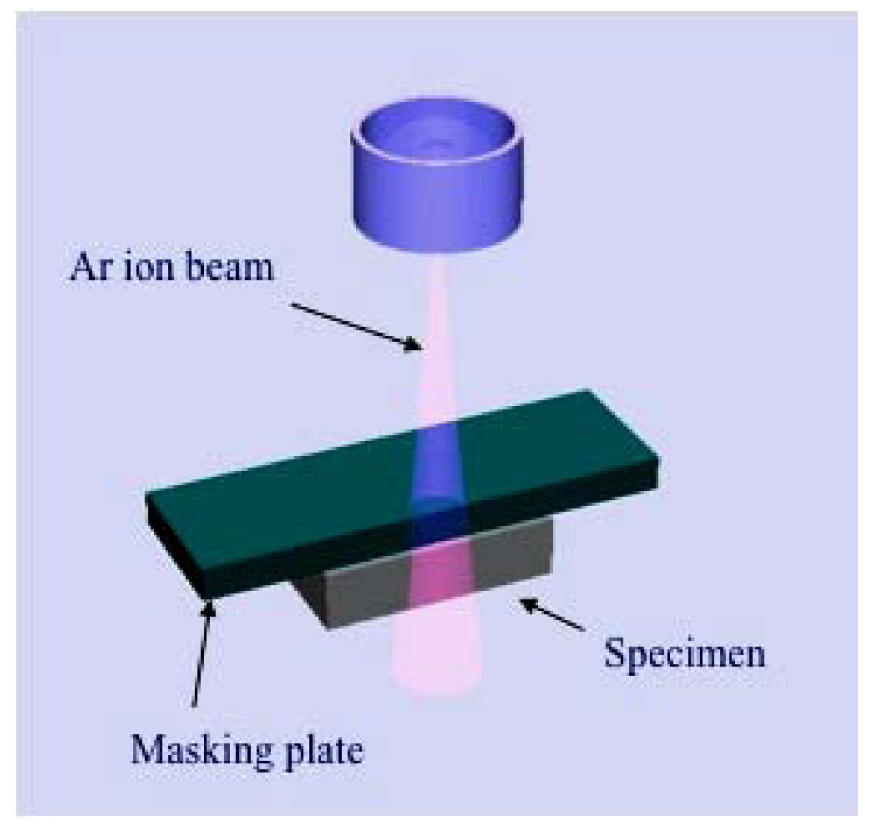

Fig. 1 Principle of CP operation.
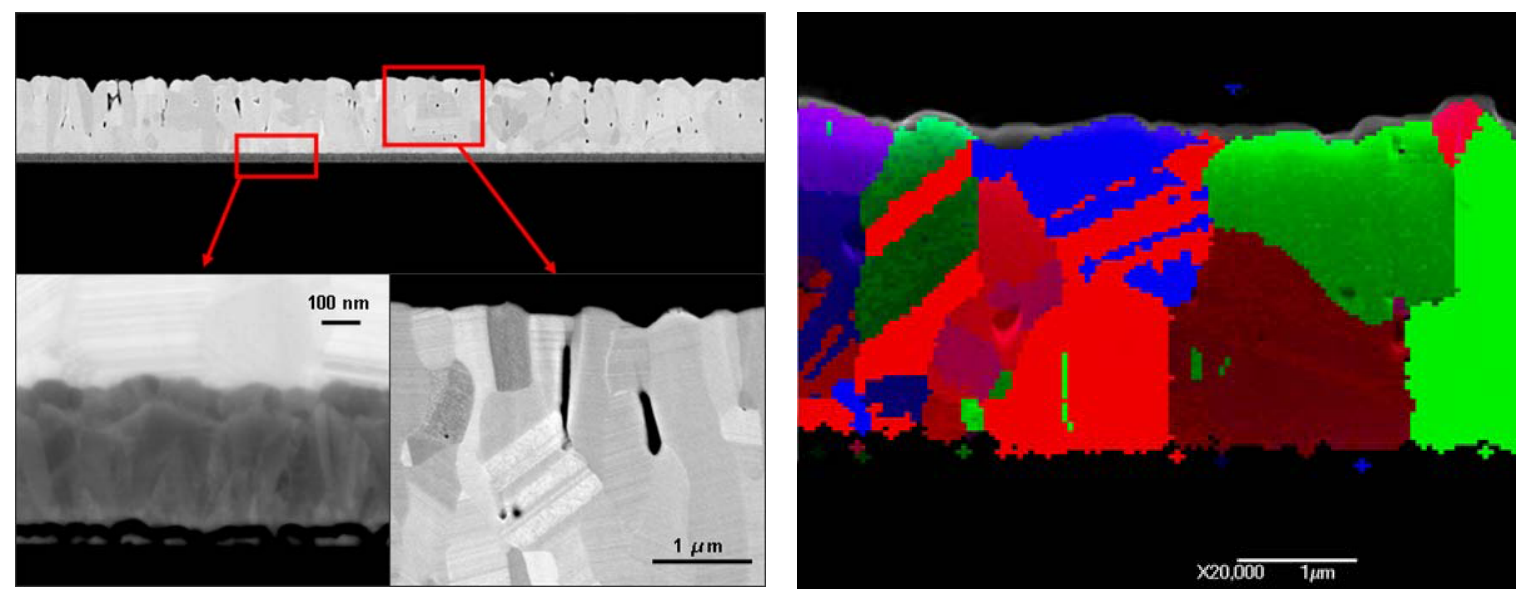

Fig. 2 Example of CdTe solar thin film on glass substrate cross-sectioned with CP. Left, BEI images of the grain structure in various layers; Right, EBSD orientation map. 